\title{
Article \\ A 3D Non-Stationary Micropolar Fluids Equations with Navier Slip Boundary Conditions
}

\author{
Cristian Duarte-Leiva ${ }^{1}$, Sebastián Lorca ${ }^{2}$ and Exequiel Mallea-Zepeda ${ }^{2, *}$ (i) \\ 1 Programa Magíster en Ciencias con Mención en Matemática, Departamento de Matemática, \\ Universidad de Tarapacá, Av. 18 de Septiembre 2222, Arica 1000000, Chile; cduartel@academicos.uta.cl \\ 2 Departamento de Matemática, Universidad de Tarapacá, Av. 18 de Septiembre 2222, Arica 1000000, Chile; \\ slorca@academicos.uta.cl \\ * Correspondence: emallea@academicos.uta.cl
}

Citation: Duarte-Leiva, C.; Lorca, S.; Mallea-Zepeda, E. A 3D

Non-Stationary Micropolar Fluids Equations with Navier Slip Boundary Conditions. Symmetry 2021, 13, 1348. https://doi.org/10.3390/sym13081348

Academic Editors: Evgenii S.

Baranovskii, Aleksey P. Zhabko and Vyacheslav Provotorov

Received: 28 June 2021

Accepted: 23 July 2021

Published: 26 July 2021

Publisher's Note: MDPI stays neutral with regard to jurisdictional claims in published maps and institutional affiliations.

Copyright: (c) 2021 by the authors. Licensee MDPI, Basel, Switzerland. This article is an open access article distributed under the terms and conditions of the Creative Commons Attribution (CC BY) license (https:// creativecommons.org/licenses/by/ $4.0 /)$.

\begin{abstract}
Micropolar fluids are fluids with microstructure and belong to a class of fluids with asymmetric stress tensor that called Polar fluids, and include, as a special case, the well-established Navier-Stokes model. In this work we study a 3D micropolar fluids model with Navier boundary conditions without friction for the velocity field and homogeneous Dirichlet boundary conditions for the angular velocity. Using the Galerkin method, we prove the existence of weak solutions and establish a Prodi-Serrin regularity type result which allow us to obtain global-in-time strong solutions at finite time.
\end{abstract}

Keywords: micropolar fluid equations; weak solutions; strong solutions

MSC: 35Q35; 76D03

\section{Introduction}

The Navier-Stokes system is a widely accepted model for describing the motion of viscous and incompressible fluids in the presence of convection. However, the NavierStokes theory is unable of describing the motion of certain fluids consisting of randomly oriented (or spherical) particles suspended in a viscous medium, where the deformation of fluid particles is ignored. A subclass of these fluids is the micropolar fluids or also called asymmetric fluids, which exhibit micro-rotational effects and micro-rotational inertia [1]. Animal blood, liquid crystals, and certain polymeric fluids are a few examples of fluids which may be represented by the mathematical model of micropolar fluids, so that it is interesting to study the behavior of such fluids. The mathematical model that describes the movement of these fluids has been introduced by Eringen in 1966 [2]. In this work we study a 3D non-stationary micropolar fluids system associated with Navier boundary conditions without friction for the velocity field and homogeneous Dirichlet boundary conditions for the microrotational velocity. Specifically, we consider $\Omega \subset \mathbb{R}^{3}$ the flow domain, which is assumed to be bounded of class $C^{2,1}$ with boundary $\Gamma:=\partial \Omega$ and $(0, T)$ a time interval, with $0<T<\infty$. Then, we analyze the following coupled non-linear system of partial differential equations, which expresses the balance of momentum, angular momentum, and mass, in the space-time region $Q:=\Omega \times(0, T)$ :

$$
\left\{\begin{aligned}
\partial_{t} \mathbf{u}+(\mathbf{u} \cdot \nabla) \mathbf{u}-\left(v+v_{r}\right) \Delta \mathbf{u}+\nabla p & =2 v_{r} \text { curlw }+\mathbf{f}, \\
\partial_{t} \mathbf{w}+(\mathbf{u} \cdot \nabla) \mathbf{w}-\left(c_{a}+c_{d}\right) \Delta \mathbf{w}-\left(c_{0}+c_{d}-c_{a}\right) \nabla \operatorname{div} \mathbf{w}+4 v_{r} \mathbf{w} & =2 v_{r} \text { curl } \mathbf{u}+\mathbf{g}, \\
\operatorname{div} \mathbf{u} & =0 .
\end{aligned}\right.
$$

Here, the unknowns are $\mathbf{u}:=\mathbf{u}(x, t) \in \mathbb{R}^{3}, \mathbf{w}:=\mathbf{w}(x, t) \in \mathbb{R}^{3}$ and $p:=p(x, t) \in \mathbb{R}$, and denote, respectively, the linear velocity field, the velocity of rotation of the particles and the pressure of the fluid at the point $(x, t) \in Q$. The functions $\mathbf{f}$ and $\mathbf{g}$ are given and 
represent external sources of linear and angular momentum of particles, respectively. The positive real constants $v, v_{r}, c_{0}, c_{a}, c_{d}$ characterize isotropic properties of the fluid. More specifically, the constant $v$ is the usual Newtonian viscosity; the constant $v_{r}$ is called the viscosity of microrotation and $c_{0}, c_{a}$ and $c_{d}$ are new viscosities related to the asymmetry of the stress tensor and satisfy $c_{0}+c_{d}>c_{a}$. For simplicity we denote $v_{1}:=v+v_{r}$, $v_{2}:=c_{a}+c_{d}$, and $v_{3}:=c_{0}+c_{d}-c_{a}$. Without loss of generality we can assume that the density of the fluid is equal to one. The symbols $\Delta, \nabla$, div and curl denote the Laplacian, gradient, divergence and rotational operators, respectively; the terms $\partial_{t} \mathbf{u}$ and $\partial_{t} \mathbf{w}$ stand for the time derivatives of $\mathbf{u}$ and $\mathbf{w}$, respectively. The $i$-th component of $(\mathbf{u} \cdot \nabla) \mathbf{u}$ and $(\mathbf{u} \cdot \nabla) \mathbf{w}$ are given, respectively, by

$$
[(\mathbf{u} \cdot \nabla) \mathbf{u}]_{i}=\sum_{j=1}^{3} u_{j} \frac{\partial u_{i}}{\partial x_{j}} \quad \text { and } \quad[(\mathbf{w} \cdot \nabla) \mathbf{u}]_{i}=\sum_{j=1}^{3} w_{j} \frac{\partial u_{i}}{\partial x_{j}}
$$

We associate to system (1) the following initial conditions

$$
\mathbf{u}(x, 0)=\mathbf{u}_{0}(x), \quad \mathbf{w}(x, 0)=\mathbf{w}_{0}(x) \quad \text { in } \Omega,
$$

and mixed boundary conditions

$$
[D(\mathbf{u}) \mathbf{n}]_{\operatorname{tang}}=\mathbf{0}, \mathbf{u} \cdot \mathbf{n}=0 \quad \text { and } \quad \mathbf{w}=\mathbf{0} \quad \text { on } \quad \Sigma:=\Gamma \times(0, T) .
$$

Here $\mathbf{n}$ denotes the outward unit normal vector to $\Gamma$. The term $[D(\mathbf{u}) \mathbf{n}]_{\operatorname{tang}}$ is the tangential component of the vector $D(\mathbf{u}) \mathbf{n}$; that is, $[D(\mathbf{u}) \mathbf{n}]_{\operatorname{tang}}:=D(\mathbf{u}) \mathbf{n}-[(D(\mathbf{u}) \mathbf{n}) \cdot \mathbf{n}] \mathbf{n}$ and $D(\mathbf{u}):=\frac{1}{2}\left(\nabla \mathbf{u}+\nabla^{T} \mathbf{u}\right)$ is twice standard symmetric part of the rate of deformation tensor. The requirements $[D(\mathbf{u}) \mathbf{n}]_{\operatorname{tang}}=\mathbf{0 ,} \mathbf{u} \cdot \mathbf{n}=0$ on $\Gamma$ are called the Navier boundary condition without friction (or also called the perfect slip boundary condition [3]) and arises in the context of free-boundary problems. The Navier boundary condition was proposed by Navier in [4] and justified as a homogenization of the non-slip condition on a rough boundary (cf. [5]). Moreover, when the boundary $\Gamma$ is flat, the fluid tends to slip over $\Gamma$ without friction and there are no boundary layers [6,7]. Hence, from the physical pointof-view, the Navier boundary condition makes more sense than the Dirichlet boundary conditions. Moreover, the mathematical analysis is more complicated, since to define a correct variational formulation of the systems (1)-(3) the classical Green identities are not applicable in this case. The above, to obtain a suitable weak formulation of the problems (1)-(3), leads us to study other results of integration by parts in the spatial variable (see Lemma 1, below). Additionally, in order to correctly control the $\mathbf{H}_{\sigma}$-norm of the Galerkin approximations in terms of the $\mathbf{L}^{2}$-norm of the deformation tensor; that is, the norm $\left\|D\left(\mathbf{u}^{m}\right)\right\|^{2}$, we must employ Korn inequality (see [8], p. 52), which is not usual. There exist some phenomena modeling of which might require the introduction of Navier boundary conditions; for instance, flow through a drain or canal with its bottom covered be sherbet of mud and pebbles, and flow of melted iron coming out from a smelting furnace, avalanche of water and rocks and blood flow in a vein of an arterial sclerosis patient (see, for instance, Fujita $[9,10])$.

From the mathematical point-of-view, the initial-value problem (1)-(2) with Dirichlet boundary conditions has been studied by several authors, and important results on existence of weak solutions and local strong solutions, large time asymptotic behavior, and general qualitative analysis, have been obtained (see, for instance, the textbook [11]). Moreover, in [12] the authors analyze the case with variable density and prove the existence of local-in-time strong solutions, using a spectral semi-Galerkin method. In addition, they prove the uniqueness of the strong solutions and some global existence results. In [13], the global existence and uniqueness of solutions through a Lagrangian approach under suitable conditions are investigated on the initial data. The same authors in [14] establish the existence of local-in-time semi-strong solutions and global-in-time strong solutions in general 3D domains. Ferreira and Villamizar-Roa [15] study the 3D generalized micropolar 
system in a space of tempered distributions and, using the Duhamel principle, prove the global existence, uniqueness, and asymptotic stability of the underlying mild solutions. Other study on the asymptotic analysis of the solutions can be see in [16]. In this work the authors obtain their results using the semigroups approach in $L^{p}$-spaces.

The purpose of this paper is to prove the existence of weak solutions of systems (1)-(3) and establish a regularity result of the Prodi-Serrin type (cf. $[17,18]$ ) that allow us obtain global-in-time strong solutions of problems (1)-(3). The literature concerning to regularity results for weak solutions of problems (1) and (2) associated with Dirichlet boundary conditions is scarce. Indeed, we can mention the works [19-24]. In [19], assuming the external forces $\mathbf{f}$ and $\mathbf{g}$ in $L^{q}\left(\mathbf{L}^{q}(\Omega)\right)$, for $q>3$, and that the pressure $p$ belongs to $L^{q}\left(\mathbf{L}^{\frac{3 q}{q+1}} \Omega\right)$, the authors improved the regularity for the weak solutions. In [23] the authors present a weak$L^{p}$ Prodi-Serrin type regularity criterion, assuming that the velocity field $\mathbf{u} \in L^{S}\left(\mathbf{L}^{r, \infty}(\Omega)\right)$, where $(s, r)$ is a Prodi-Serrin pair and $\mathbf{L}^{r, \infty}(\Omega)$ denotes the weak- $\mathbf{L}^{r}$ space; that is, the space of measurable functions $\mathbf{f}$, such that $\|\mathbf{f}\|_{\mathbf{L}^{r, \infty}}=\sup \left\{\alpha \cdot d_{\mathbf{f}}(\alpha)^{1 / r}: \alpha>0\right\}$ is finite, with $d_{\mathbf{f}}=m(\{x \in \Omega:|f(x)|>\alpha\})$. In the recent analysis developed by Ragusa and $\mathrm{Wu}$ [24], the authors establish a regularity criterion in terms of the one partial derivative of the velocity; that is, they assume that $\partial_{3} \mathbf{u} \in L^{\frac{2}{1-r}}\left(B_{\infty, \infty}^{-r}\right)$, with $0<r<1$, and $B_{\infty, \infty}^{-r}$ is a Besov space with negative order of regularity $-r$, and prove that the weak solution $(\mathbf{u}, \mathbf{w})$ is also strong (see [22], for positive indices). In this work, we establish a regularity criterion imposing only the condition that $\mathbf{u} \in L^{S}\left(\mathbf{L}^{r}(\Omega)\right)$, where $(s, r)$ is a Prodi-Serrin pair $\left(r \in(3, \infty), s \in(2, \infty)\right.$ with $\frac{2}{s}+\frac{3}{r} \leq 1$, see Theorem 2$)$.

We recall that when $\mathbf{w}=\mathbf{0}$, systems (1)-(3) is reduced to the Navier-Stokes equations with Navier boundary conditions, for which there is a good amount of studies. In [25] the authors study the inhomogeneous (variable density) 3D Navier-Stokes system and prove the existence of weak solutions using the Galerkin method. Additionally, they analyze the inviscid limits of solutions to strong solutions of the corresponding inhomogeneous Euler system, as the viscosity $v$ goes to 0 , under suitable regularity assumptions on external force $\mathbf{f}$ and the initial velocity $\mathbf{u}_{0}$ and that the initial density is separated from zero; that is, $\rho_{0} \geq \rho_{*}>0$. Mulone and Salemi [26] prove the existence of generalized solutions in an either bounded or exterior domain. Moreover, they prove the existence of periodic solutions under assumptions that the flow domain is bounded and the external forces are periodic in time. These results are extended by the same authors in [27], considering the case of non-homogeneous Navier boundary conditions for a bounded domain. Other results related with exterior domains can be consulted in [28]; moreover, the authors obtain $L^{p}-L^{q}$ estimates for the Stokes system and this result leads to global-in-time existence for the Navier-Stokes systems with small initial data in $\mathbf{L}^{n}(\Omega)$, where $n$ is the spatial dimension. The stationary Navier-Stokes system with Navier boundary condition has been analyzed in [29-31]. Solonnikov and Šcadilov [30], assuming that the density of the fluid is constant, prove the existence of weak solutions and in [29] the authors generalize these results using $L^{p}$-theory. In [31], the case of variable density in 2D domains and, using the stream-Frolov approach for the density, the existence of weak solutions is studied and is proven. The steady-state problem related to systems (1)-(3) has been studied in [32,33]. In [32], the 3D case with constant density is analyzed and proves the existence and uniqueness of weak solutions using the Galerkin method. In [33], the authors study the 2D case with variable density and, using the stream-Frolov approach for the density, prove the existence of weak solutions. Studies related with low-concentrated aqueous polymer systems subject to the Navier slip boundary conditions have been developed by Baranovskii [34]. In this work the author proved the existence of global-in-time weak solutions and, assuming additional regularity for the weak solutions, established some uniqueness results.

The outline of this paper is a follows: In Section 2, we fix the notation, introduce the functional spaces to be used throughout this work and give the concept of weak solutions of systems (1)-(3). In Section 3 we prove the existence of weak solutions applying the Galerkin method. Finally, in Section 4 we present a regularity result, of Prodi-Serrin type, under which a weak solution of (1)-(3) is also a strong solution and unique (see Theorem 2). 


\section{Preliminaries}

In this section, some notations will be introduced. The Lebesgue space $L^{p}(\Omega)$, $1 \leq p \leq \infty$, with norm denoted by $\|\cdot\|_{L^{p}}$ will be used. In particular, the $L^{2}$-norm and its inner product will be denoted by $\|\cdot\|$ and $(\cdot, \cdot)$, respectively. The usual Sobolev spaces $W^{m, p}(\Omega)=\left\{u \in L^{p}(\Omega):\left\|\partial^{\alpha} u\right\|_{L^{p}}<\infty, \forall|\alpha| \leq m\right\}$, with norm denoted by $\|\cdot\|_{W^{m, p}}$ is considered. When $p=2$, it is established $H^{m}(\Omega):=W^{m, 2}(\Omega)$ denoting the respective norm by $\|\cdot\|_{H^{m}}$. The function spaces of vector-valued spaces will be denoted by bold script; for instance, $\mathbf{H}^{1}(\Omega), \mathbf{L}^{p}(\Omega)$, and so on. Additionally, we will use the following solenoidal Hilbert space $\mathbf{H}_{\sigma}:=\left\{\mathbf{u} \in \mathbf{H}^{1}(\Omega): \operatorname{div} \mathbf{u}=0\right.$ and $\mathbf{u} \cdot \mathbf{n}=0$ on $\left.\Gamma\right\}$. The space $\mathbf{H}_{\sigma}$ is endowed with the usual norm and inner product of $\mathbf{H}^{1}(\Omega)$. Additionally, we consider the space $\mathbf{H}_{0}^{1}(\Omega):=\left\{\mathbf{u} \in \mathbf{H}^{1}(\Omega): \mathbf{u}=\mathbf{0}\right.$ on $\left.\Gamma\right\}$, which is a Hilbert space with the inner-product $(\mathbf{u}, \mathbf{v})_{\mathbf{H}_{0}^{1}}:=(\nabla \mathbf{u}, \nabla \mathbf{v})$ and the norm $\|\mathbf{u}\|_{\mathbf{H}_{0}^{1}}:=\|\nabla \mathbf{u}\|$.

Additionally, as usual, we define $\mathcal{V}:=\left\{\mathbf{u} \in C_{0}^{\infty}(\Omega): \operatorname{div} \mathbf{u}=0\right\}$ and the space

$$
\mathbf{H}:=\text { the closure of } \mathcal{V} \text { in } \mathbf{L}^{2}(\Omega) .
$$

The space $\mathbf{H}$ is characterized by [35]:

$$
\mathbf{H}=\left\{\mathbf{u} \in \mathbf{L}^{2}(\Omega): \operatorname{div} \mathbf{u}=0 \text { and } \mathbf{u} \cdot \mathbf{n}=0 \text { on } \Gamma\right\} .
$$

If $X$ is a Banach space, $L^{p}(X)$ indicates the space of valued functions in $X$ defined on the interval $[0, T]$ that are integrable in the Bochner sense, and its norm will be denoted by $\|\cdot\|_{L^{p}(X)}$. For simplicity, one defines $L^{p}(Q):=L^{p}\left(0, T ; \mathbf{L}^{p}(\Omega)\right)$ if $p \neq \infty$ and its norm by $\|\cdot\|_{L^{p}(Q)}$. In the case $p=\infty, L^{\infty}(Q)$ means $L^{\infty}((0, T) \times \Omega)$ and its norm is denoted by $\|\cdot\|_{L^{\infty}(Q)}$. It is denoted by $C([0, T] ; X)$ the space of continuous functions from $[0, T]$ into a Banach space $X$, whose norm is given by $\|\cdot\|_{C(X)}$. The topological dual space of a Banach space $X$ will be denoted by $X^{\prime}$, and the duality for a pair $X$ and $X^{\prime}$ by $\langle\cdot, \cdot\rangle_{X^{\prime}}$ or simply by $\langle\cdot, \cdot\rangle$ unless this leads to ambiguity. In particular $\mathbf{H}^{-1}(\Omega)$ will denote the dual space of $\mathbf{H}_{0}^{1}(\Omega)$ and $\mathbf{H}_{\sigma}^{\prime}$ the dual of $\mathbf{H}_{\sigma}$. Moreover, the letters $C, C_{0}, C_{1}, \ldots$, denote positive constants, independent of $\mathbf{u}$ and $\mathbf{w}$, but its value may be change from line to line.

In order to define a weak formulation of micropolar fluids systems (1)-(3), we consider the Stokes operator $A:=-P \Delta$ with domain

$$
D(A)=\left\{\mathbf{u} \in \mathbf{H}^{2}(\Omega) \cap \mathbf{H}_{\sigma}:[D(\mathbf{u}) \mathbf{n}]_{\operatorname{tang}}=\mathbf{0} \text { on } \Gamma\right\},
$$

where $P: \mathbf{L}^{2}(\Omega) \rightarrow \mathbf{H}$ is the Leray projector and the strongly elliptic Lamé operator $L:=-v_{2} \Delta-v_{3} \nabla$ div, with domain $D(L)=\mathbf{H}^{2}(\Omega) \cap \mathbf{H}_{0}^{1}(\Omega)$. We recall that operator $A$ establish a one-to-one correspondence of $D(A)$ onto $\mathbf{H}_{\sigma}$, is positive-definite on $D(A)$, self-adjoint and its inverse operator is compact (see $[26,27])$. Thus, its spectrum is discrete, positive and of finite multiplicity, the eigenvalues $\lambda_{k}$ converges to $\infty$, the eigenfunctions $\left\{\boldsymbol{\phi}_{k}\right\}_{k=1}^{\infty}$ are orthogonal and complete in $\mathbf{H}_{\sigma}$ and satisfy $\left[D\left(\boldsymbol{\phi}_{k}\right) \mathbf{n}\right]_{\operatorname{tang}}=\mathbf{0}$ on $\Gamma$.

Additionally, we introduce the trilinear forms $b_{1}: \mathbf{H}_{\sigma} \times \mathbf{H}_{\sigma} \times \mathbf{H}_{\sigma} \rightarrow \mathbb{R}$ and $b_{2}$ : $\mathbf{H}_{\sigma} \times \mathbf{H}^{1}(\Omega) \times \mathbf{H}_{0}^{1}(\Omega) \rightarrow \mathbb{R}$ by

$$
b_{1}\left(\mathbf{u}_{1}, \mathbf{v}_{1}, \mathbf{w}_{1}\right)=\left(\left(\mathbf{u}_{1} \cdot \nabla\right) \mathbf{v}_{1}, \mathbf{w}_{1}\right), \quad b_{2}\left(\mathbf{u}_{2}, \mathbf{v}_{2}, \mathbf{w}_{2}\right)=\left(\left(\mathbf{u}_{2} \cdot \nabla\right) \mathbf{v}_{2}, \mathbf{w}_{2}\right),
$$

which satisfy the following properties (see, for instance, [36]):

$$
\begin{aligned}
b_{1}\left(\mathbf{u}_{1}, \mathbf{v}_{1}, \mathbf{w}_{1}\right) & =-b_{1}\left(\mathbf{u}_{1}, \mathbf{w}_{1}, \mathbf{v}_{1}\right) \quad \forall \mathbf{u}_{1}, \mathbf{v}_{1}, \mathbf{w}_{1} \in \mathbf{H}_{\sigma}, \\
b_{1}\left(\mathbf{u}_{1}, \mathbf{v}_{1}, \mathbf{v}_{1}\right) & =0 \quad \forall \mathbf{u}_{1}, \mathbf{v}_{1} \in \mathbf{H}_{\sigma}, \\
b_{2}\left(\mathbf{u}_{2}, \mathbf{v}_{2}, \mathbf{w}_{2}\right) & =-b_{2}\left(\mathbf{u}_{2}, \mathbf{w}_{2}, \mathbf{v}_{2}\right) \quad \forall \mathbf{u}_{2} \in \mathbf{H}_{\sigma}, \mathbf{v}_{2} \in \mathbf{H}^{1}(\Omega), \mathbf{w}_{2} \in \mathbf{H}_{0}^{1}(\Omega), \\
b_{2}\left(\mathbf{u}_{2}, \mathbf{v}_{2}, \mathbf{v}_{2}\right) & =0 \quad \forall \mathbf{u}_{2} \in \mathbf{H}_{\sigma}, \mathbf{v}_{2} \in \mathbf{H}_{0}^{1}(\Omega) .
\end{aligned}
$$


These trilinear forms induce the bilinear operators $B_{1}: \mathbf{H}_{\sigma} \times \mathbf{H}_{\sigma} \rightarrow \mathbf{H}_{\sigma}^{\prime}$ and $B_{2}$ : $\mathbf{H}_{\sigma} \times \mathbf{H}^{1}(\Omega) \rightarrow \mathbf{H}^{-1}(\Omega)$ defined by

$$
\left\{\begin{array}{l}
\left\langle B_{1}\left(\mathbf{u}_{1}, \mathbf{v}_{1}\right), \mathbf{w}_{1}\right\rangle_{\mathbf{H}_{\sigma}^{\prime}}=b_{1}\left(\mathbf{u}_{1}, \mathbf{v}_{1}, \mathbf{w}_{1}\right) \forall \mathbf{w}_{1} \in \mathbf{H}_{\sigma} \\
\left\langle B_{2}\left(\mathbf{u}_{2}, \mathbf{v}_{2}\right), \mathbf{w}_{2}\right\rangle_{\mathbf{H}^{-1}}=b_{2}\left(\mathbf{u}_{2}, \mathbf{v}_{2}, \mathbf{w}_{2}\right) \forall \mathbf{w}_{2} \in \mathbf{H}_{0}^{1}(\Omega) .
\end{array}\right.
$$

Remark 1. From the classical interpolation inequality in $3 D$ domains $\|\mathbf{u}\|_{\mathbf{L}^{4}} \leq C\|\mathbf{u}\|^{1 / 4}\|\mathbf{u}\|_{\mathbf{H}^{1}}^{3 / 4}$ for all $\mathbf{u} \in \mathbf{H}^{1}(\Omega)$, Poincaré inequality, and properties (5) and (7) we can deduce that

$$
B_{1}(\mathbf{u}, \mathbf{u}) \in \mathbf{L}^{4 / 3}\left(\mathbf{H}_{\sigma}^{\prime}\right) \text { and } B_{2}(\mathbf{u}, \mathbf{v}) \in \mathbf{L}^{4 / 3}\left(\mathbf{H}^{-1}(\Omega)\right) .
$$

Moreover, since $\mathbf{w}=\mathbf{0}$ on $\Gamma$ it holds Pcurl $\mathbf{w}=\operatorname{curl} \mathbf{w}$. In fact, let $\operatorname{curl} \mathbf{w}=\widetilde{\mathbf{w}}+\nabla q$ with $\widetilde{\mathbf{w}} \in \mathbf{H}$ and $q \in H^{1}(\Omega)$ the Helmholtz decomposition of curl $\mathbf{w}$ in $\mathbf{L}^{2}(\Omega)$ (see [35]). Then,

$$
\|\nabla q\|^{2}+(\widetilde{\mathbf{w}}, \nabla q)=\int_{\Gamma}(\mathbf{w} \times \mathbf{n}) \cdot \nabla q=0 .
$$

Thus, $\nabla q=\mathbf{0}$ and, consequently, $\widetilde{\mathbf{w}}=P \operatorname{curl} \mathbf{w}=\operatorname{curl} \mathbf{w}$.

Then, applying the Leray projector to $(1)_{1}$ and considering the above notations, we can rewrite systems (1)-(3) in the following equivalent system

$$
\left\{\begin{aligned}
\partial_{t} \mathbf{u}+v_{1} A \mathbf{u}+P B_{1}(\mathbf{u}, \mathbf{u}) & =2 v_{r} \operatorname{curl} \mathbf{w}+P \mathbf{f} \text { in } Q, \\
\partial_{t} \mathbf{w}+L \mathbf{w}+B_{2}(\mathbf{u}, \mathbf{w})+4 v_{r} \mathbf{w} & =2 v_{r} \operatorname{curl} \mathbf{u}+\mathbf{g} \text { in } Q, \\
\mathbf{u}(x, 0) & =\mathbf{u}_{0}(x), \mathbf{w}(x, 0)=\mathbf{w}_{0}(x) \text { in } \Omega, \\
{[D(\mathbf{u}) \mathbf{n}]_{\text {tang }}=\mathbf{0}, \mathbf{u} \cdot \mathbf{n} } & =0, \quad \mathbf{w}=\mathbf{0} \quad \text { on } \Sigma .
\end{aligned}\right.
$$

Lemma 1 ([30]). Let $(\mathbf{u}, \mathbf{v}) \in \mathbf{H}^{2}(\Omega) \times \mathbf{H}^{1}(\Omega)$ with divergence free and tangent to the boundary. Then,

$$
\begin{aligned}
-\int_{\Omega} \Delta \mathbf{u} \cdot \mathbf{v} & =2 \int_{\Omega} D(\mathbf{u}): D(\mathbf{v})-2 \int_{\Gamma}[D(\mathbf{u}) \mathbf{n}]_{\operatorname{tang}} \cdot \mathbf{v} \\
& =2(D(\mathbf{u}), D(\mathbf{v}))-2 \int_{\Gamma}[D(\mathbf{u}) \mathbf{n}]_{\operatorname{tang}} \cdot \mathbf{v} .
\end{aligned}
$$

Now, we establish the concept of weak solutions of systems (1)-(3) (equivalently problem (11)).

Definition 1. Let $(\mathbf{f}, \mathbf{g}) \in L^{2}(Q) \times L^{2}(Q)$ and the initial data $\left(\mathbf{u}_{0}, \mathbf{w}_{0}\right) \in \mathbf{H} \times \mathbf{L}^{2}(\Omega)$. We say that the pair $(\mathbf{u}, \mathbf{w})$ is a weak solution of $(11)$ in $(0, T)$, if

$$
\begin{aligned}
\mathbf{u} \in \mathbf{W}_{\mathbf{u}} & :=\left\{\mathbf{u} \in L^{\infty}(\mathbf{H}) \cap L^{2}\left(\mathbf{H}_{\sigma}\right): \partial_{t} \mathbf{u} \in L^{4 / 3}\left(\mathbf{H}_{\sigma}^{\prime}\right)\right\} \\
\mathbf{w} \in \mathbf{W}_{\mathbf{w}} & :=\left\{\mathbf{w} \in L^{\infty}\left(\mathbf{L}^{2}(\Omega)\right) \cap L^{2}\left(\mathbf{H}_{0}^{1}(\Omega)\right): \partial_{t} \mathbf{w} \in L^{4 / 3}\left(\mathbf{H}^{-1}(\Omega)\right)\right\},
\end{aligned}
$$

and satisfies the following variational formulation

$$
\left\{\begin{aligned}
\left\langle\partial_{t} \mathbf{u}, \mathbf{v}\right\rangle_{\mathbf{H}_{\sigma}^{\prime}}+v_{1}\langle A \mathbf{u}, \mathbf{v}\rangle_{\mathbf{H}_{\sigma}^{\prime}}+\left\langle P B_{1}(\mathbf{u}, \mathbf{u}), \mathbf{v}\right\rangle_{\mathbf{H}_{\sigma}^{\prime}} & =2 v_{r}(\operatorname{curl} \mathbf{w}, \mathbf{v})+(P \mathbf{f}, \mathbf{v}) \\
\left\langle\partial_{t} \mathbf{w}, \mathbf{z}\right\rangle_{\mathbf{H}^{-1}}+\langle L \mathbf{w}, \mathbf{z}\rangle_{\mathbf{H}^{-1}}+\left\langle B_{2}(\mathbf{u}, \mathbf{w}), \mathbf{z}\right\rangle_{\mathbf{H}^{-1}}+4 v_{r}(\mathbf{w}, \mathbf{z}) & =2 v_{r}(\operatorname{curl} \mathbf{u}, \mathbf{z})+(\mathbf{g}, \mathbf{z}) \\
\mathbf{u}(x, 0) & =\mathbf{u}_{0}(x) \text { in } \mathbf{H} \\
\mathbf{w}(x, 0) & =\mathbf{w}_{0}(x) \text { in } \mathbf{L}^{2}(\Omega)
\end{aligned}\right.
$$

for all $(\mathbf{v}, \mathbf{z}) \in \mathbf{H}_{\sigma} \times \mathbf{H}_{0}^{1}(\Omega)$ and almost every $t \in(0, T)$. 
Remark 2. From Lemma 1, the Equations $(14)_{1}$ and $(14)_{2}$ are understood in the following sense

$$
\begin{gathered}
\int_{0}^{t}\left\langle\partial_{t} \mathbf{u}, \mathbf{v}\right\rangle_{\mathbf{H}_{\sigma}^{\prime}}+2 v_{1} \int_{0}^{t}(D(\mathbf{u}), D(\mathbf{v}))+\int_{0}^{t}\left\langle P B_{1}(\mathbf{u}, \mathbf{u}), \mathbf{v}\right\rangle_{\mathbf{H}_{\sigma}^{\prime}} \\
=2 v_{r} \int_{0}^{t}(\operatorname{curl} \mathbf{w}, \mathbf{v})+\int_{0}^{t}(P \mathbf{f}, \mathbf{v}) \quad \forall \mathbf{v} \in \mathbf{H}_{\sigma}, \\
\int_{0}^{t}\left\langle\partial_{t} \mathbf{w}, \mathbf{z}\right\rangle_{\mathbf{H}^{-1}}+\int_{0}^{t}\left(L^{1 / 2} \mathbf{w}, L^{1 / 2} \mathbf{z}\right)+\int_{0}^{t}\left\langle B_{2}(\mathbf{u}, \mathbf{w}), \mathbf{z}\right\rangle_{\mathbf{H}^{-1}}+4 v_{r} \int_{0}^{t}(\mathbf{w}, \mathbf{z}) \\
=2 v_{r} \int_{0}^{t}(\operatorname{curl} \mathbf{u}, \mathbf{z})+\int_{0}^{t}(\mathbf{g}, \mathbf{z}) \quad \forall \mathbf{z} \in \mathbf{H}_{0}^{1}(\Omega),
\end{gathered}
$$

for almost every $t \in(0, T)$. Here, $L^{1 / 2}:=v_{2}^{1 / 2} \nabla+v_{3}^{1 / 2}$ div.

\section{Existence of Weak Solutions of Problems (1)-(3)}

This section is dedicated to prove the existence of weak solutions of problems (1)-(3). The proof is carry out using the Galerkin method. Specifically we will prove the following result.

Theorem 1. (Existence) Let $(\mathbf{f}, \mathbf{g}) \in L^{2}(Q) \times L^{2}(Q)$ and $\left(\mathbf{u}_{0}, \mathbf{w}_{0}\right) \in \mathbf{H} \times \mathbf{L}^{2}(\Omega)$. There exists at least one weak solution of system (11) in sense of Definition 1.

Proof. To prove the existence of solution of problem (11) we will use the Galerkin method. Step 1: Construction of the approximate solutions.

Let $\left\{\left(\mathbf{v}_{i}, \mathbf{z}_{i}\right)\right\}_{i=1}^{\infty}$ be a sequence of orthonormal functions, such that its linear hull is dense in the product space $\mathbf{H}_{\sigma} \times \mathbf{H}_{0}^{1}(\Omega)$. Now, for each $m \in \mathbb{N}$, we consider the spaces $\mathbf{H}_{\sigma}^{m}$ and $\mathbf{H}^{m}$, which are spanned by $\left\{\mathbf{v}_{1}, \ldots, \mathbf{v}_{m}\right\}$ and $\left\{\mathbf{z}_{1}, \ldots, \mathbf{z}_{m}\right\}$, respectively. Then, we define the approximate solution $\left(\mathbf{u}^{m}, \mathbf{w}^{m}\right) \in \mathbf{H}_{\sigma}^{m} \times \mathbf{H}^{m}$ of problem (11) as follows:

$$
\mathbf{u}^{m}(x, t):=\sum_{i=1}^{m} \lambda_{i m}(t) \mathbf{v}_{i}(x), \quad \mathbf{w}^{m}(x, t):=\sum_{i=1}^{m} \eta_{i m}(t) \mathbf{z}_{i}(x),
$$

satisfying

$$
\begin{gathered}
\left\langle\partial_{t} \mathbf{u}^{m}, \mathbf{v}\right\rangle_{\mathbf{H}_{\sigma}^{\prime}}+2 v_{1}\left(D\left(\mathbf{u}^{m}\right), D(\mathbf{v})\right)+\left\langle P B_{1}\left(\mathbf{u}^{m}, \mathbf{u}^{m}\right), \mathbf{v}\right\rangle_{\mathbf{H}_{\sigma}^{\prime}} \\
=2 v_{r}\left(\operatorname{curl} \mathbf{w}^{m}, \mathbf{v}\right)+\left(P_{m} \mathbf{f}, \mathbf{v}\right) \quad \forall \mathbf{v} \in \mathbf{H}_{\sigma}, \\
\left\langle\partial_{t} \mathbf{w}^{m}, \mathbf{z}\right\rangle_{\mathbf{H}^{-1}}+\left(L^{1 / 2} \mathbf{w}^{m}, L^{1 / 2} \mathbf{z}\right)+\left\langle B_{2}\left(\mathbf{u}^{m}, \mathbf{w}^{m}\right), \mathbf{z}\right\rangle_{\mathbf{H}^{-1}}+4 v_{r}\left(\mathbf{w}^{m}, \mathbf{z}\right) \\
\quad=2 v_{r}\left(\operatorname{curl} \mathbf{u}^{m}, \mathbf{z}\right)+(\mathbf{g}, \mathbf{z}) \quad \forall \mathbf{z} \in \mathbf{H}_{0}^{1}(\Omega), \\
\mathbf{u}^{m}(x, 0)=P_{m} \mathbf{u}_{0}^{m}, \quad \mathbf{w}^{m}(x, 0)=\widetilde{P}_{m} \mathbf{w}_{0}^{m},
\end{gathered}
$$

where $P_{m}$ and $\widetilde{P}_{m}$ denote, respectively, the orthogonal projection from $\mathbf{H}$ onto $\mathbf{H}_{\sigma}^{m}$ and from $\mathbf{L}^{2}(\Omega)$ onto $\mathbf{H}^{m}$.

We observe that the systems (17)-(19) can be regarded as Cauchy problem for a firstorder ordinary differential system, where the unknowns are the functions $\lambda_{i m}(\cdot)$ and $\eta_{i m}(\cdot)$. Therefore, the classical existence and uniqueness theory for ordinary differential systems can be applied; thus, we deduce that, for each $m \in \mathbb{N}$, there exists a unique pair $\left(\mathbf{u}^{m}, \mathbf{w}^{m}\right)$ solutions of (17)-(19) on a time interval $\left[0, T_{m}\right]$. If $T_{m}<T$, then $\left\|\left(\mathbf{u}^{m}, \mathbf{w}^{m}\right)\right\|_{\mathbf{H}_{\sigma} \times \mathbf{H}_{0}^{1}}^{2}$ must be tend to $\infty$, as $t$ goes to $T_{m}$; then, the uniform estimates show that this does not happen, and, thus, $T_{m}=T$ (for more details, see ([35], Chapter 3)).

Step 2: A priori estimates. 
The aim in this step is to obtain uniform estimates of $\left(\mathbf{u}^{m}, \mathbf{w}^{m}\right)$ and $\left(\partial_{t} \mathbf{u}^{m}, \partial_{t} \mathbf{w}^{m}\right)$. Indeed, making $\mathbf{v}=\mathbf{u}^{m}$ in (17) and $\mathbf{z}=\mathbf{w}^{m}$ in (18), then adding the respective equations, and taking into account properties (6) and (8), we can obtain

$$
\begin{aligned}
\frac{1}{2} \frac{d}{d t}\left(\left\|\mathbf{u}^{m}\right\|^{2}+\left\|\mathbf{w}^{m}\right\|^{2}\right)+ & 2 v_{1}\left\|D\left(\mathbf{u}^{m}\right)\right\|^{2}+v_{2}\left\|\mathbf{w}^{m}\right\|_{\mathbf{H}_{0}^{1}}^{2}+4 v_{r}\left\|\mathbf{w}^{m}\right\|^{2} \\
\leq & 2 v_{r}\left|\left(\operatorname{curl} \mathbf{w}^{m}, \mathbf{u}^{m}\right)\right|+2 v_{r}\left|\left(\operatorname{curl} \mathbf{u}^{m}, \mathbf{w}^{m}\right)\right| \\
& +\left|\left(P_{m} \mathbf{f}, \mathbf{u}^{m}\right)\right|+\left|\left(\mathbf{g}, \mathbf{w}^{m}\right)\right| .
\end{aligned}
$$

Now, we will bound the right-hand side of (20). In fact, applying the Hölder, Poincaré, and Young inequalities we obtain

$$
\begin{aligned}
\left|\left(P_{m} \mathbf{f}, \mathbf{u}^{m}\right)\right| & \leq C\|\mathbf{f}\|\left\|\mathbf{u}^{m}\right\| \leq \frac{C}{v_{1}}\|\mathbf{f}\|^{2}+v_{1}\left\|\mathbf{u}^{m}\right\|^{2}, \\
\left|\left(\mathbf{g}, \mathbf{w}^{m}\right)\right| & \leq\|\mathbf{g}\|\left\|\mathbf{w}^{m}\right\| \leq \frac{C}{v_{r}}\|\mathbf{g}\|^{2}+4 v_{r}\left\|\mathbf{w}^{m}\right\|^{2} .
\end{aligned}
$$

Furthermore, since $\left(\operatorname{curl} \mathbf{w}^{m}, \mathbf{u}^{m}\right)=\left(\operatorname{curl} \mathbf{u}^{m}, \mathbf{w}^{m}\right)$ and $\|\operatorname{curl} \mathbf{z}\| \leq \sqrt{2}\|\nabla \mathbf{z}\|$, for each vector field $\mathbf{z} \in \mathbf{H}^{1}(\Omega)$; then from the Hölder and Young inequalities we have

$$
\begin{aligned}
2 v_{r}\left|\left(\operatorname{curl} \mathbf{w}^{m}, \mathbf{u}^{m}\right)\right| & \leq 2 v_{r}\left\|\operatorname{curl}^{\mathbf{w}^{m}}\right\|\left\|\mathbf{u}^{m}\right\| \leq 2 \sqrt{2} v_{r}\left\|\mathbf{w}^{m}\right\|_{\mathbf{H}_{0}^{1}}\left\|\mathbf{u}^{m}\right\| \\
& \leq \frac{v_{2}}{4}\left\|\mathbf{w}^{m}\right\|_{\mathbf{H}_{0}^{1}}^{2}+\frac{v_{r}^{2} C}{v_{2}}\left\|\mathbf{u}^{m}\right\|^{2}, \\
2 v_{r}\left|\left(\operatorname{curl} \mathbf{u}^{m}, \mathbf{w}^{m}\right)\right| & =2 v_{r}\left|\left(\operatorname{curl} \mathbf{w}^{m}, \mathbf{u}^{m}\right)\right| \leq 2 \sqrt{2} v_{r}\left\|\mathbf{w}^{m}\right\|_{\mathbf{H}_{0}^{1}}\left\|\mathbf{u}^{m}\right\| \\
& \leq \frac{v_{2}}{4}\left\|\mathbf{w}^{m}\right\|_{\mathbf{H}_{0}^{1}}^{2}+\frac{v_{r}^{2} C}{v_{2}}\left\|\mathbf{u}^{m}\right\|^{2} .
\end{aligned}
$$

Therefore, replacing (21)-(24) in (20) and adding $2 v_{1}\left\|\mathbf{u}^{m}\right\|^{2}$ to both sides in the resulting inequality, we can obtain

$$
\begin{aligned}
& \frac{d}{d t}\left(\left\|\mathbf{u}^{m}\right\|^{2}+\left\|\mathbf{w}^{m}\right\|^{2}\right)+4 v_{1}\left(\left\|D\left(\mathbf{u}^{m}\right)\right\|^{2}+\left\|\mathbf{u}^{m}\right\|^{2}\right)+v_{2}\left\|\mathbf{w}^{m}\right\|_{\mathbf{H}_{0}^{1}}^{2} \\
& \leq\left(6 v_{1}+\frac{4 v_{r}^{2}}{v_{2}} C\right)\left\|\mathbf{u}^{m}\right\|^{2}+C\left(\|\mathbf{f}\|^{2}+\|\mathbf{g}\|^{2}\right) \\
& \leq\left(6 v_{1}+\frac{4 v_{r}^{2}}{v_{2}} C\right)\left(\left\|\mathbf{u}^{m}\right\|^{2}+\left\|\mathbf{w}^{m}\right\|^{2}\right)+C\left(\|\mathbf{f}\|^{2}+\|\mathbf{g}\|^{2}\right) .
\end{aligned}
$$

From the Korn inequality (see, for instance, ([8], p. 52)) we have that there exists a positive constant $C_{K}$, such that $C_{K}\left\|\mathbf{u}^{m}\right\|_{\mathbf{H}_{\sigma}}^{2} \leq\left(\left\|D\left(\mathbf{u}^{m}\right)\right\|^{2}+\left\|\mathbf{u}^{m}\right\|^{2}\right)$. Thus, from (25) we obtain

$$
\begin{aligned}
& \frac{d}{d t}\left(\left\|\mathbf{u}^{m}\right\|^{2}+\left\|\mathbf{w}^{m}\right\|^{2}\right)+4 v_{1} C_{K}\left\|\mathbf{u}^{m}\right\|_{\mathbf{H}_{\sigma}}^{2}+v_{2}\left\|\mathbf{w}^{m}\right\|_{\mathbf{H}_{0}^{1}}^{2} \\
& \quad \leq\left(6 v_{1}+\frac{4 v_{r}^{2}}{v_{2}} C\right)\left(\left\|\mathbf{u}^{m}\right\|^{2}+\left\|\mathbf{w}^{m}\right\|^{2}\right)+C\left(\|\mathbf{f}\|^{2}+\|\mathbf{g}\|^{2}\right) .
\end{aligned}
$$

Moreover, recalling that $P_{m}: \mathbf{H} \rightarrow \mathbf{H}_{\sigma}^{m}$ and $\widetilde{P}_{m}: \mathbf{L}^{2}(\Omega) \rightarrow \mathbf{H}^{m}$ are the orthogonal projections (see (19)) we have

$$
\begin{aligned}
\left\|\mathbf{u}^{m}(0)\right\|^{2} & =\left\|P_{m} \mathbf{u}_{0}^{m}\right\|^{2} \leq\left\|\mathbf{u}_{0}\right\|^{2}, \\
\left\|\mathbf{w}^{2}(0)\right\|^{2} & =\left\|\widetilde{P}_{m} \mathbf{w}_{0}^{m}\right\|^{2} \leq\left\|\mathbf{w}_{0}\right\|^{2} .
\end{aligned}
$$

Then, from (26) and Gronwall lemma deduce the following estimate 


$$
\begin{aligned}
\left\|\mathbf{u}^{m}\right\|_{L^{\infty}(\mathbf{H})}^{2}+\left\|\mathbf{w}^{m}\right\|_{L^{\infty}\left(\mathbf{L}^{2}\right)}^{2} & \leq C \exp \left(6 v_{1} T+\frac{4 v_{r}^{2}}{v_{2}} T\right)\left(\left\|\mathbf{u}^{m}(0)\right\|^{2}+\left\|\mathbf{w}^{m}(0)\right\|^{2}+\|\mathbf{f}\|_{L^{2}(Q)}^{2}+\|\mathbf{g}\|_{L^{2}(Q)}^{2}\right) \\
& \leq C \exp \left(6 v_{1} T+\frac{4 v_{r}^{2}}{v_{2}} T\right)\left(\left\|\mathbf{u}_{0}\right\|^{2}+\left\|\mathbf{w}_{0}\right\|^{2}+\|\mathbf{f}\|_{L^{2}(Q)}^{2}+\|\mathbf{g}\|_{L^{2}(Q)}^{2}\right)
\end{aligned}
$$

Hence, integrating over $[0, T]$ in (26) and using (27) we conclude that there exists a positive constant $C_{1}$, independent of $m$, such that

$$
\left\|\mathbf{u}^{m}\right\|_{L^{\infty}(\mathbf{H}) \cap L^{2}\left(\mathbf{H}_{\sigma}\right)}+\left\|\mathbf{w}^{m}\right\|_{L^{\infty}\left(\mathbf{L}^{2}\right) \cap L^{2}\left(\mathbf{H}_{0}^{1}\right)} \leq C_{1}
$$

Consequently,

$$
\left\{\begin{array}{l}
\left\{\mathbf{u}^{m}\right\}_{m \geq 1} \text { is bounded in } L^{\infty}(\mathbf{H}) \cap L^{2}\left(\mathbf{H}_{\sigma}\right) \\
\left\{\mathbf{w}^{m}\right\}_{m \geq 1} \text { is bounded in } L^{\infty}\left(\mathbf{L}^{2}(\Omega)\right) \cap L^{2}\left(\mathbf{H}_{0}^{1}(\Omega)\right) .
\end{array}\right.
$$

Now, in order to obtain uniform estimates for $\partial_{t} \mathbf{u}^{m}$ and $\partial_{t} \mathbf{w}^{m}$, we observe that from $(14)_{1}$ and $(14)_{2},(17)$ and (18) we have

$$
\begin{aligned}
\left\langle\partial_{t} \mathbf{u}^{m}, \mathbf{v}\right\rangle_{\mathbf{H}_{\sigma}^{\prime}} & =\left\langle 2 v_{r} \operatorname{curl} \mathbf{w}^{m}+P_{m} \mathbf{f}-v_{1} A \mathbf{u}^{m}-P B_{1}\left(\mathbf{u}^{m}, \mathbf{u}^{m}\right), \mathbf{v}\right\rangle_{\mathbf{H}_{\sigma^{\prime}}^{\prime}} \\
\left\langle\partial_{t} \mathbf{w}^{m}, \mathbf{z}\right\rangle_{\mathbf{H}^{-1}} & =\left\langle 2 v_{r} \operatorname{curl}^{m}+\mathbf{g}-L \mathbf{w}^{m}-B_{2}\left(\mathbf{u}^{m}, \mathbf{w}^{m}\right)-4 v_{r} \mathbf{w}^{m}, \mathbf{z}\right\rangle_{\mathbf{H}^{-1}},
\end{aligned}
$$

which jointly to (10) and (28) implies that

$$
\begin{aligned}
\left\|\partial_{t} \mathbf{u}^{m}\right\|_{L^{4 / 3}\left(\mathbf{H}_{\sigma}^{\prime}\right)}= & \left\|2 v_{r} \operatorname{curl} \mathbf{w}^{m}+P_{m} \mathbf{f}-v_{1} A \mathbf{u}^{m}-B_{1}\left(\mathbf{u}^{m}, \mathbf{u}^{m}\right)\right\|_{L^{4 / 3}\left(\mathbf{H}_{\sigma}^{\prime}\right)} \\
\leq & 2 v_{r}\left\|\operatorname{curl} \mathbf{w}^{m}\right\|_{L^{4 / 3}\left(\mathbf{H}_{\sigma}^{\prime}\right)}+\left\|P_{m} \mathbf{f}\right\|_{L^{4 / 3}\left(\mathbf{H}_{\sigma}^{\prime}\right)}+v_{1}\left\|A \mathbf{u}^{m}\right\|_{L^{4 / 3}\left(\mathbf{H}_{\sigma}^{\prime}\right)} \\
& +\left\|P B_{1}\left(\mathbf{u}^{m}, \mathbf{u}^{m}\right)\right\|_{L^{4 / 3}\left(\mathbf{H}_{\sigma}^{\prime}\right)} \\
\leq & C_{2} \\
\left\|\partial_{t} \mathbf{w}^{m}\right\|_{L^{4 / 3}\left(\mathbf{H}^{-1}\right)}= & \left\|2 v_{r} \operatorname{curl} \mathbf{u}^{m}+\mathbf{g}-L \mathbf{w}^{m}-B_{2}\left(\mathbf{u}^{m}, \mathbf{w}^{m}\right)-4 v_{r} \mathbf{w}^{m}\right\|_{L^{4 / 3}\left(\mathbf{H}^{-1}\right)} \\
\leq & 2 v_{r}\left\|\operatorname{curl}^{m} \mathbf{u}^{m}\right\|_{L^{4 / 3}\left(\mathbf{H}^{-1}\right)}+\|\mathbf{g}\|_{L^{4 / 3}\left(\mathbf{H}^{-1}\right)}+\|L \mathbf{w}\|_{L^{4 / 3}\left(\mathbf{H}^{-1}\right)} \\
& +\left\|B_{2}\left(\mathbf{u}^{m}, \mathbf{w}^{m}\right)\right\|_{L^{4 / 3}\left(\mathbf{H}^{-1}\right)}+4 v_{r}\left\|\mathbf{w}^{m}\right\|_{L^{4 / 3}\left(\mathbf{H}^{-1}\right)} \\
\leq & C_{3} .
\end{aligned}
$$

Thus, from (29) and (30) we conclude that

$$
\left\{\left(\partial_{t} \mathbf{u}^{m}, \partial_{t} \mathbf{w}^{m}\right)\right\}_{m \geq 1} \text { is bounded in } L^{4 / 3}\left(\mathbf{H}_{\sigma}^{\prime}\right) \times L^{4 / 3}\left(\mathbf{H}^{-1}(\Omega)\right) .
$$

Step 3: Passage to the limit.

From (28) and (31) we deduce that there exists a limit element $(\mathbf{u}, \mathbf{w}) \in \mathbf{W}_{\mathbf{u}} \times \mathbf{W}_{\mathbf{w}}$, such that for some subsequence of $\left\{\left(\mathbf{u}^{m}, \mathbf{w}^{m}\right)\right\}_{m \geq 1}$, still denoted by $\left\{\left(\mathbf{u}^{m}, \mathbf{w}^{m}\right)\right\}_{m \geq 1}$, the following convergences hold, as $m$ goes to $\infty$ :

$$
\left\{\begin{aligned}
\mathbf{u}^{m} & \rightarrow \text { u weakly in } L^{2}\left(\mathbf{H}_{\sigma}\right) \text { and weakly* in } L^{\infty}(\mathbf{H}), \\
\mathbf{w}^{m} & \rightarrow \mathbf{w} \text { weakly in } L^{2}\left(\mathbf{H}_{0}^{1}(\Omega)\right) \text { and weakly in } L^{\infty}\left(\mathbf{L}^{2}(\Omega)\right), \\
\left(\partial_{t} \mathbf{u}^{m}, \partial_{t} \mathbf{w}^{m}\right) & \rightarrow\left(\partial_{t} \mathbf{u}, \partial_{t} \mathbf{w}\right) \text { weakly* in } L^{4 / 3}\left(\mathbf{H}_{\sigma}^{\prime}\right) \times L^{4 / 3}\left(\mathbf{H}^{-1}(\Omega)\right) .
\end{aligned}\right.
$$

Moreover, from (32), the Aubin-Lions lemma (see ([37], Theorem 5.1, p. 58)) and a Simon compactness result in Bochner spaces (see ([38], Corollary 4)), we have

$$
\left\{\begin{aligned}
\mathbf{u}^{m} & \rightarrow \mathbf{u} \text { strongly in } L^{2}(\mathbf{H}) \cap C\left([0, T] ; \mathbf{H}_{\sigma}^{\prime}\right) \\
\mathbf{w}^{m} & \rightarrow \mathbf{w} \text { strongly in } L^{2}(Q) \cap C\left([0, T] ; \mathbf{H}^{-1}(\Omega)\right) ;
\end{aligned}\right.
$$


which imply that $\left(\mathbf{u}^{m}(0), \mathbf{w}^{m}(0)\right)$ converges to $(\mathbf{u}(0), \mathbf{w}(0))$ in $\mathbf{H}_{\sigma}^{\prime} \times \mathbf{H}^{-1}(\Omega)$, and considering that $\left(\mathbf{u}^{m}(0), \mathbf{w}^{m}(0)\right)=\left(P_{m} \mathbf{u}_{0}^{m}, \widetilde{P}_{m} \mathbf{w}_{0}^{m}\right)$, for each $m$, and that $\left(P_{m} \mathbf{u}_{0}^{m}, \widetilde{P}_{m} \mathbf{w}_{0}^{m}\right) \rightarrow\left(\mathbf{u}_{0}, \mathbf{w}_{0}\right)$ strongly in $\mathbf{H} \times \mathbf{L}^{2}(\Omega)$; from the uniqueness of the limit we deduce the identification $(\mathbf{u}(0), \mathbf{w}(0))=\left(\mathbf{u}_{0}, \mathbf{w}_{0}\right)$ in the space $\mathbf{H} \times \mathbf{L}^{2}(\Omega)$, which are the initial conditions given in $(14)_{3}$ and $(14)_{4}$.

Therefore, the convergences (32) and (33), and a standard procedure allows us pass to the limit in (17)-(19), as $m$ goes to $\infty$; and thus, we conclude that $(\mathbf{u}, \mathbf{w})$ is a weak solution of system (11).

\section{Strong Solutions}

In this section, we present a Prodi-Serrin type regularity result that allow us obtain global-in-time strong solutions of system (11).

Firstly, we will establish the concept of strong solution of problem (11).

Definition 2. (Strong solutions) Let $(\mathbf{f}, \mathbf{g}) \in L^{2}(Q) \times L^{2}(Q)$ and $\left(\mathbf{u}_{0}, \mathbf{w}_{0}\right) \in \mathbf{H}_{\sigma} \times \mathbf{H}_{0}^{1}(\Omega)$. We say that the pair $(\mathbf{u}, \mathbf{w})$ is a strong solution of system (11) in the time interval $(0, T)$, if

$$
\begin{aligned}
\mathbf{u} \in \mathbf{S}_{\mathbf{u}}:=\left\{\mathbf{u} \in L^{\infty}\left(\mathbf{H}_{\sigma}\right) \cap L^{2}\left(\mathbf{H}^{2}(\Omega)\right): \partial_{t} \mathbf{u} \in L^{2}(\mathbf{H})\right\}, \\
\mathbf{w} \in \mathbf{S}_{\mathbf{w}}:=\left\{\mathbf{w} \in L^{\infty}\left(\mathbf{H}_{0}^{1}(\Omega)\right) \cap L^{2}\left(\mathbf{H}^{2}(\Omega)\right): \partial_{t} \mathbf{w} \in L^{2}(Q)\right\},
\end{aligned}
$$

satisfies pointwisely a.e. $(x, t) \in Q$ the problem

$$
\left\{\begin{aligned}
\partial_{t} \mathbf{u}+v_{1} A \mathbf{u}+P B_{1}(\mathbf{u}, \mathbf{u}) & =2 v_{r} \operatorname{curl} \mathbf{w}+P \mathbf{f} \\
\partial_{t} \mathbf{w}+L \mathbf{w}+B_{2}(\mathbf{u}, \mathbf{w})+4 v_{r} \mathbf{w} & =2 v_{r} \operatorname{curl} \mathbf{u}+\mathbf{g}
\end{aligned}\right.
$$

jointly to initial and boundary conditions $(11)_{3}$ and $(11)_{4}$, respectively.

Thus, we have the following result.

Theorem 2. Let $(\mathbf{u}, \mathbf{w}) \in \mathbf{W}_{\mathbf{u}} \times \mathbf{W}_{\mathbf{w}}$ be a weak solution of (11). If, in addition $\left(\mathbf{u}_{0}, \mathbf{w}_{0}\right) \in$ $\mathbf{H}_{\sigma} \times \mathbf{H}_{0}^{1}(\Omega)$ and

$$
\mathbf{u} \in L^{\frac{2 r}{r-3}}\left(\mathbf{L}^{r}(\Omega)\right),
$$

with $r \in(3, \infty)$. Then, $(\mathbf{u}, \mathbf{w})$ is the unique strong solution of system (11) in sense of Definition 2.

Proof. We separate te proof in two steps.

Regularity: We fix $\mathbf{w} \in \mathbf{W}_{\mathbf{w}}$; then, first we improve the regularity for $\mathbf{u}$ and after for $\mathbf{w}$. In fact, we perform formally the estimates that strong solution $(\mathbf{u}, \mathbf{w})$ must satisfy. An exhaustive proof would be performed using Galerkin approximation for each pair of functions $(\mathbf{u}, \mathbf{w})$.

Testing (36) $)_{1}$ by $A \mathbf{u}$ and applying Lemma 1 we have

$$
\frac{1}{2} \frac{d}{d t}\|D(\mathbf{u})\|^{2}+v_{1}\|A \mathbf{u}\|^{2} \leq\left|\left\langle P B_{1}(\mathbf{u}, \mathbf{u}), A \mathbf{u}\right\rangle_{\mathbf{H}_{\sigma}^{\prime}}\right|+2 v_{r}|(\operatorname{curl} \mathbf{w}, A \mathbf{u})|+|(P \mathbf{f}, A \mathbf{u})| .
$$

Now we will bound the right-hand side of (38). In fact, from the Hölder inequality we obtain

$$
\left|\left\langle P B_{1}(\mathbf{u}, \mathbf{u}), A \mathbf{u}\right\rangle_{\mathbf{H}_{\sigma}^{\prime}}\right| \leq C\|\mathbf{u}\|_{\mathbf{L}^{r}}\|\nabla \mathbf{u}\|_{\mathbf{L}^{s}}\|A \mathbf{u}\|,
$$

with $\frac{1}{r}+\frac{1}{s}=\frac{1}{2}$. Additionally, we observe that from the Gagliardo-Nirenberg interpolation inequality (see, for instance, [39]) and taking into account the equivalent norms $\|\mathbf{u}\|_{\mathbf{H}^{2}} \equiv\|A \mathbf{u}\|$ (cf. [26]), we can deduce

$$
\|\nabla \mathbf{u}\|_{L^{s}} \leq C\|\nabla \mathbf{u}\|^{\frac{6-s}{2 s}}\|A \mathbf{u}\|^{\frac{3 s-6}{2 s}},
$$


which, jointly to (39) and Young inequality, imply

$$
\begin{aligned}
\left|\left\langle P B_{1}(\mathbf{u}, \mathbf{u}), A \mathbf{u}\right\rangle_{\mathbf{H}_{\sigma}^{\prime}}\right| & \leq C\|\mathbf{u}\|_{\mathbf{L}^{r}}\|\nabla \mathbf{u}\|^{\frac{6-s}{2 s}}\|A \mathbf{u}\|^{\frac{5 s-6}{2 s}} \\
& \leq C\|\mathbf{u}\|_{\mathbf{L}^{r}}^{\frac{4 s}{6-s}}\|\nabla \mathbf{u}\|^{2}+\frac{v_{1}}{6}\|A \mathbf{u}\|^{2} .
\end{aligned}
$$

Moreover, since $\frac{1}{r}+\frac{1}{s}=\frac{1}{2}$, we have $s=\frac{2 r}{r-2}$; thus, $\frac{4 s}{6-s}=\frac{2 r}{r-3}$. Consequently, we obtain the following estimate

$$
\left|\left\langle P B_{1}(\mathbf{u}, \mathbf{u}), A \mathbf{u}\right\rangle_{\mathbf{H}_{\sigma}^{\prime}}\right| \leq C\|\mathbf{u}\|_{\mathbf{L}^{r}}^{\frac{2 r}{r-3}}\|\nabla \mathbf{u}\|^{2}+\frac{v_{1}}{6}\|A \mathbf{u}\|^{2} .
$$

On the other hand, using again the Hölder and Young inequalities we have

$$
\begin{aligned}
2 v_{r}|(\operatorname{curl} \mathbf{w}, A \mathbf{u})| & \leq 2 v_{r}\|\operatorname{curl} \mathbf{w}\|\|A \mathbf{u}\| \leq C\|\operatorname{curl} \mathbf{w}\|^{2}+\frac{v_{1}}{6}\|A \mathbf{u}\|^{2} \\
& \leq C\|\mathbf{w}\|_{\mathbf{H}_{0}^{1}}^{2}+\frac{v_{1}}{6}\|A \mathbf{u}\|^{2}, \\
|(P \mathbf{f}, A \mathbf{u})| & \leq C\|\mathbf{f}\|\|A \mathbf{u}\| \leq C\|\mathbf{f}\|^{2}+\frac{v_{1}}{6}\|A \mathbf{u}\|^{2} .
\end{aligned}
$$

Then, replacing (40)-(42) in (38) we obtain

$$
\begin{aligned}
\frac{d}{d t}\|D(\mathbf{u})\|^{2}+v_{1}\|A \mathbf{u}\|^{2} & \leq C\|\mathbf{u}\|_{\mathbf{L}^{r}}^{\frac{2 r}{r-3}}\|\nabla \mathbf{u}\|^{2}+C\left(\|\mathbf{w}\|_{\mathbf{H}_{0}^{1}}^{2}+\|\mathbf{f}\|^{2}\right) \\
& \leq C\|\mathbf{u}\|_{\mathbf{L}^{r}}^{\frac{2 r}{r-3}}\|D \mathbf{u}\|^{2}+C\left(\|\mathbf{w}\|_{\mathbf{H}_{0}^{1}}^{2}+\|\mathbf{f}\|^{2}\right) .
\end{aligned}
$$

Therefore, from (43), Gronwall lemma, the equivalence $\|\mathbf{u}\|_{\mathbf{H}^{2}} \equiv\|A \mathbf{u}\|$ and taking into account the hypothesis (37), we deduce that

$$
\mathbf{u} \in L^{\infty}\left(\mathbf{H}_{\sigma}\right) \cap L^{2}\left(\mathbf{H}^{2}(\Omega)\right) \hookrightarrow L^{10}(Q) .
$$

Furthermore, from (44) and interpolating we have $\nabla \mathbf{u} \in L^{\infty}\left(\mathbf{L}^{2}(\Omega)\right) \cap L^{2}\left(\mathbf{H}^{1}(\Omega)\right) \hookrightarrow$ $L^{10 / 3}(Q)$, which jointly to (44) imply

$$
B_{1}(\mathbf{u}, \mathbf{u}) \in L^{5 / 2}(Q) .
$$

Hence, using that $A \mathbf{u} \in L^{2}(\mathbf{H})$, from (45) and (36) 1 we conclude that $\partial_{t} \mathbf{u} \in L^{2}(\mathbf{H})$. Thus, $\mathbf{u} \in \mathbf{S}_{\mathbf{u}}$.

Now, we will improve the regularity for $\mathbf{w}$. Testing $(36)_{2}$ by $-\Delta \mathbf{w}$ we have

$$
\frac{1}{2} \frac{d}{d t}\|\mathbf{w}\|_{\mathbf{H}_{0}^{1}}^{2}+(L \mathbf{w},-\Delta \mathbf{w})+4 v_{r}\|\mathbf{w}\|_{\mathbf{H}_{0}^{1}}^{2} \leq\left|\left\langle B_{2}(\mathbf{u}, \mathbf{w}), \Delta \mathbf{w}\right\rangle_{\mathbf{H}^{-1}}\right|+2 v_{r}|(\operatorname{curl} \mathbf{u}, \Delta \mathbf{w})|+|(\mathbf{g}, \Delta \mathbf{w})| .
$$

From the Hölder and Young inequalities we have

$$
\begin{aligned}
2 v_{r}|(\operatorname{curl} \mathbf{u}, \Delta \mathbf{w})| & \leq 2 v_{r}\|\operatorname{curl} \mathbf{u}\|\|\Delta \mathbf{w}\| \leq 2 \sqrt{2} v_{r}\|\nabla \mathbf{u}\|\|\Delta \mathbf{w}\| \\
& \leq C\|D(\mathbf{u})\|^{2}+\frac{v_{2}}{6}\|\Delta \mathbf{w}\|, \\
|(\mathbf{g}, \Delta \mathbf{w})| & \leq\|\mathbf{g}\|\|\Delta \mathbf{w}\| \leq C\|\mathbf{g}\|^{2}+\frac{v_{2}}{6}\|\Delta \mathbf{w}\| .
\end{aligned}
$$

Additionally, arguing as in (40) we can obtain

$$
\left|\left\langle B_{2}(\mathbf{u}, \mathbf{w}), \Delta \mathbf{w}\right\rangle_{\mathbf{H}^{-1}}\right| \leq C\|\mathbf{u}\|_{\mathbf{L}^{r}}^{\frac{2 r}{r-3}}\|\mathbf{w}\|_{\mathbf{H}_{0}^{1}}^{2}+\frac{v_{2}}{6}\|\Delta \mathbf{w}\|^{2} .
$$


On the other hand, taking into account that the operator $L \mathbf{w}=-v_{2} \Delta \mathbf{w}-v_{3} \nabla \operatorname{div} \mathbf{w}$ is strongly elliptic, there exists a positive constant $\widetilde{C}:=\widetilde{C}\left(v_{2}, v_{3}, \Gamma\right)$, such that (see [23])

$$
(L \mathbf{w},-\Delta \mathbf{w}) \geq v_{2}\|\Delta \mathbf{w}\|^{2}-\widetilde{C}\|\mathbf{w}\|_{\mathbf{H}_{0}^{1}}^{2} .
$$

Thus, using the estimates (47)-(50) in (46) we have

$\frac{1}{2} \frac{d}{d t}\|\mathbf{w}\|_{\mathbf{H}_{0}^{1}}^{2}+\frac{v_{2}}{2}\|\Delta \mathbf{w}\|^{2}+4 v_{r}\|\mathbf{w}\|_{\mathbf{H}_{0}^{1}}^{2} \leq C\|D(\mathbf{u})\|^{2}+C\|\mathbf{g}\|^{2}+\widetilde{C}\|\mathbf{w}\|_{\mathbf{H}_{0}^{1}}^{2}+C\|\mathbf{u}\| \mathbf{L}^{\frac{2 r}{r-3}}\|\mathbf{w}\|_{\mathbf{H}_{0}^{1}}^{2} ;$ hence,

$$
\frac{d}{d t}\|\mathbf{w}\|_{\mathbf{H}_{0}^{1}}^{2}+C\|\mathbf{w}\|_{\mathbf{H}^{2}}^{2} \leq C\|D(\mathbf{u})\|^{2}+C\left(\|\mathbf{u}\|_{\mathbf{L}^{r}}^{\frac{2 r}{r-3}}+1\right)\|\mathbf{w}\|_{\mathbf{H}_{0}^{1}}^{2}+C\|\mathbf{g}\|^{2} .
$$

Therefore, from (51), Gronwall lemma and hypothesis (37) we deduce that

$$
\mathbf{w} \in L^{\infty}\left(\mathbf{H}_{0}^{1}(\Omega)\right) \cap L^{2}\left(\mathbf{H}^{2}(\Omega)\right) \hookrightarrow L^{10}(Q) ;
$$

and, since $\nabla \mathbf{w} \in L^{\infty}\left(\mathbf{L}^{2}(\Omega)\right) \cap L^{2}\left(\mathbf{H}^{1}(\Omega)\right) \hookrightarrow L^{10 / 3}(Q)$ we have $B_{2}(\mathbf{u}, \mathbf{w}) \in L^{5 / 2}(Q)$. Then, using that $\Delta \mathbf{w} \in L^{2}(Q)$, from (36) 2 we deduce that $\partial_{t} \mathbf{w} \in L^{2}(Q)$. Thus, we conclude that $\mathbf{w} \in \mathbf{S}_{\mathbf{w}}$.

Uniqueness: We will apply a classical comparison argument. Indeed, let $\left(\mathbf{u}^{1}, \mathbf{w}^{1}\right),\left(\mathbf{u}^{2}, \mathbf{w}^{2}\right) \in \mathbf{S}_{\mathbf{u}} \times \mathbf{S}_{\mathbf{w}}$ two possible solutions of problem (36). Then, subtracting equations in (36) for $\left(\mathbf{u}^{1}, \mathbf{w}^{1}\right)$ and $\left(\mathbf{u}^{2}, \mathbf{w}^{2}\right)$, and making $\mathbf{u}:=\mathbf{u}^{1}-\mathbf{u}^{2}$ and $\mathbf{w}:=\mathbf{w}^{1}-\mathbf{w}^{2}$ we can obtain the following system

$$
\left\{\begin{aligned}
\partial_{t} \mathbf{u}+v_{1} A \mathbf{u}+P\left(B_{1}\left(\mathbf{u}^{1}, \mathbf{u}\right)+B_{1}\left(\mathbf{u}, \mathbf{u}^{2}\right)\right) & =2 v_{r} \operatorname{curl} \mathbf{w} \text { in } Q \\
\partial_{t} \mathbf{w}+L \mathbf{w}+B_{2}\left(\mathbf{u}^{1}, \mathbf{w}\right)+B_{2}\left(\mathbf{u}, \mathbf{w}^{2}\right) & =2 v_{r} \operatorname{curl} \mathbf{u} \text { in } Q \\
\mathbf{u}(x, 0)=\mathbf{0}, \mathbf{w}(x, 0) & =\mathbf{0} \text { in } \Omega \\
{[D(\mathbf{u}) \mathbf{n}]_{\operatorname{tang}}=\mathbf{0}, \mathbf{u} \cdot \mathbf{n}=0, \mathbf{w} } & =\mathbf{0} \text { on } \Sigma
\end{aligned}\right.
$$

Testing (52) by $\mathbf{u}$ and (52) 2 by $\mathbf{w}$, and using (6) and (8) we have

$$
\begin{aligned}
& \frac{1}{2} \frac{d}{d t}\left(\|\mathbf{u}\|^{2}+\|\mathbf{w}\|^{2}\right)+2 v_{1}\|D(\mathbf{u})\|^{2}+v_{2}\|\mathbf{w}\|_{\mathbf{H}_{0}^{1}}^{2} \\
& \leq\left|\left(P B_{1}\left(\mathbf{u}, \mathbf{u}^{2}\right), \mathbf{u}\right)\right|+\left|\left(B_{2}\left(\mathbf{u}, \mathbf{w}^{2}\right), \mathbf{w}\right)\right|+2 v_{r}|(\operatorname{curl} \mathbf{w}, \mathbf{u})|+2 v_{r}|(\operatorname{curl} \mathbf{u}, \mathbf{w})| .
\end{aligned}
$$

From the Hölder, Young, and Poincaré inequalities, arguing as in (40) and taking into account the 3D interpolation estimate $\|\mathbf{u}\|_{\mathbf{L}^{4}} \leq C\|\mathbf{u}\|^{1 / 4}\|D(\mathbf{u})\|^{3 / 4}$ we obtain

$$
\begin{aligned}
\left|\left(P B_{1}\left(\mathbf{u}, \mathbf{u}^{2}\right), \mathbf{u}\right)\right| & =\left|\left(P B_{1}(\mathbf{u}, \mathbf{u}), \mathbf{u}^{2}\right)\right| \leq C\|\mathbf{u}\|_{\mathbf{L}^{s}}\|\nabla \mathbf{u}\|\left\|\mathbf{u}^{2}\right\|_{\mathbf{L}^{r}} \\
& \leq \frac{v_{1}}{2}\|D \mathbf{u}\|^{2}+C\|\mathbf{u}\|^{2}\left\|\mathbf{u}^{2}\right\|_{\mathbf{L}^{r}}^{\frac{2 r}{r-3}}, \\
\left|\left(B_{2}\left(\mathbf{u}, \mathbf{w}^{2}\right), \mathbf{w}\right)\right| & =\left|\left(B_{2}(\mathbf{u}, \mathbf{w}), \mathbf{w}^{2}\right)\right| \leq\|\mathbf{u}\|_{\mathbf{L}^{4}}\|\mathbf{w}\|_{\mathbf{H}_{0}^{1}}\left\|\mathbf{w}^{2}\right\|_{\mathbf{L}^{4}} \\
& \leq \frac{v_{1}}{2}\|D(\mathbf{u})\|^{2}+\frac{v_{2}}{6}\|\mathbf{w}\|_{\mathbf{H}_{0}^{1}}^{2}+C\|\mathbf{u}\|^{2}\left\|\mathbf{w}^{2}\right\|_{\mathbf{H}_{0}^{1}}^{8} \\
2 v_{r}|(\operatorname{curl} \mathbf{w}, \mathbf{u})| & \leq 2 v_{r}\|\operatorname{curl} \mathbf{w}\|\|\mathbf{u}\| \leq 2 \sqrt{2} v_{r}\|\mathbf{w}\|_{\mathbf{H}_{0}^{1}}\|\mathbf{u}\| \\
& \leq \frac{v_{2}}{6}\|\mathbf{w}\|_{\mathbf{H}_{0}^{1}}^{2}+C\|\mathbf{u}\|^{2}, \\
2 v_{r}|(\operatorname{curl} \mathbf{u}, \mathbf{w})| & =2 v_{r}|(\operatorname{curl} \mathbf{w}, \mathbf{u})| \leq \frac{v_{2}}{6}\|\mathbf{w}\|_{\mathbf{H}_{0}^{1}}^{2}+C\|\mathbf{u}\|^{2} .
\end{aligned}
$$


Replacing (54)-(57) into (53), adding $2 v_{1}\|\mathbf{u}\|^{2}$ to both sides in the resulting inequality and applying the Korn inequality, we can obtain the following estimate

$$
\frac{d}{d t}\left(\|\mathbf{u}\|^{2}+\|\mathbf{w}\|^{2}\right)+3 v_{1} C_{K}\|\mathbf{u}\|_{\mathbf{H}_{\sigma}}^{2}+v_{2}\|\mathbf{w}\|_{\mathbf{H}_{0}^{1}}^{2} \leq C\left(\left\|\mathbf{u}^{2}\right\|_{\mathbf{L}^{r}}^{\frac{2 r}{r-3}}+\left\|\mathbf{w}^{2}\right\|_{\mathbf{H}_{0}^{1}}^{8}\right)\|\mathbf{u}\|^{2} .
$$

Therefore, considering hypothesis (37) we have that $\mathbf{u}^{2} \in L^{\frac{2 r}{r-3}}\left(\mathbf{L}^{r}(\Omega)\right)$ and that $\mathbf{w}^{2} \in L^{\infty}\left(\mathbf{H}_{0}^{1}(\Omega)\right)$, from Gronwall lemma, (58) and using that $\left(\mathbf{u}_{0}, \mathbf{w}_{0}\right)=(\mathbf{0}, \mathbf{0})$, we deduce that $\mathbf{u}=\mathbf{w}=\mathbf{0}$, and the uniqueness follows.

Remark 3. In Theorem 2, we observe that for $r \in(3, \infty)$ the exponent $\frac{2 r}{r-3} \in(2, \infty)$. Thus, making $s:=\frac{2 r}{r-3}$ we can reformulated Theorem 2 under assumption $\mathbf{u} \in L^{s}\left(\mathbf{L}^{r}(\Omega)\right)$, with

$$
\frac{2}{s}+\frac{3}{r} \leq 1, r \in(3, \infty), s \in(2, \infty) .
$$

A pair $(r, s)$ satisfying $(59)$ is called a Prodi-Serrin pair $[17,18]$.

\section{Conclusions}

In this paper we have analyzed a 3D non-stationary micropolar fluids equations considering Navier boundary conditions without friction (perfect slip boundary conditions) for the velocity field and homogeneous Dirichlet boundary conditions for the angular velocity. The main results obtained are: existence of global-in-time weak solutions at finite time and a Prodi-Serrin type regularity criterion, imposed only for the velocity field, which allow us obtain global-in-time strong solutions and, as consequence, their uniqueness. The existence of weak solutions is obtained by applying the Galerkin method and energy estimates and the improvement of their regularity is obtained through energy estimates and interpolation inequalities in Sobolev spaces (Gagliardo-Nirenberg, Korn, among others).

This work was inspired by the study developed by professors Loayza and RojasMedar [23], in which the authors obtain a weak- $L^{p}$ Prodi-Serrin type regularity criterion for the micropolar fluids system with homogeneous Dirichlet boundary conditions for the velocity field and angular velocity, assuming that both external forces $\mathbf{f}$ and $\mathbf{g}$ belong to weak- $\mathbf{L}^{r}$ spaces. Here we have improved the regularity of the weak solutions without the need to change the classical spaces for $\mathbf{f}$ and $\mathbf{g}$; that is, we have kept $(\mathbf{f}, \mathbf{g}) \in L^{2}(Q) \times L^{2}(Q)$. The key to this has been to carefully employ the Gagliardo-Nirenberg interpolation inequality (see estimates (39) and (40)). Finally, for future research, we will consider the following topics:

- The analysis of the Boussinesq system with Navier boundary conditions;

- The study of optimal control problems related to systems (1)-(3) and find the existence of global optimal solutions and derive the respective optimality system;

- $\quad$ The numerical analysis of systems (1)-(3).

Author Contributions: C.D.-L., S.L., E.M.-Z. contributed to each part of this study equally. All authors have read and agreed to the published version of the manuscript.

Funding: This research received no external funding.

Institutional Review Board Statement: Not applicable.

Informed Consent Statement: Not applicable.

Data Availability Statement: Not applicable.

Acknowledgments: C.D.-L. has been supported by postgraduate scholarship Apoyo Institucional para Magíster Académicos de la Universidad de Tarapacá, Universidad de Tarapacá, Chile. S.L. has been supported by Proyecto UTA-Mayor 4744-19, Universidad de Tarapacá, Chile. E.M.-Z. has been supported by Proyecto UTA-Mayor 4751-20, Universidad de Tarapacá, Chile. Moreover, the authors would like to thank the anonymous referees for their kind and helpful remarks and comments. 
Conflicts of Interest: The authors declare no conflict of interest.

\section{References}

1. Eringen, A.C. Simple microfluids. Int. J. Engrg. Sci. 1964, 2, 205-217. [CrossRef]

2. Eringen, A.C. Theory of micropolar fluids. J. Math. Mech. 1966, 16, 1-16. [CrossRef]

3. Le Roux, C. Existence and uniqueness of the flow of second-grade fluids with slip boundary conditions. Arch. Ration. Mech. Anal. 1999, 148, 309-356. [CrossRef]

4. Navier, C.L. Sur le lois du mouvement des fluides. Mem. Acad. R. Sci. Inst. France 1823, 6, 389-416.

5. Jägger, W.; Mikelić, A. On the roughness-induced effective boundary conditions for an incompressible viscous flow. J. Differ. Equ. 2001, 170, 96-122. [CrossRef]

6. Coron, J.-M. On the controllability of the 2-D incompressible Navier-Stokes equations with Navier slip boundary condition. ESAIM Control Optim. Cal. Var. 1996, 1, 35-75. [CrossRef]

7. Coron, F. Derivation of slip boundary conditions for the Navier-Stokes system from the Boltzmann equation. J. Statical Phys. 1989, 54, 829-857. [CrossRef]

8. Raviart, P.A.; Thomas, J.M. Introduction À l'analyse Numérique de Èquations aux Dérivées Partielles. In Collection Mathématiques Appliquées pour la Maîtrise; Masson: Paris, France, 1983.

9. Fujita, H. A mathematical analysis of motions of viscous incompressible fluid under leak or slip boundary conditions. Rest. Inst. Math. Sci. 1994, 888, 199-216.

10. Fujita, H. A coherent analysis of Stokes flow under boundary conditions of friction type. J. Comp. Appl. Math. 2002, 149, 57-69. [CrossRef]

11. Lukaszewicz, G. Micropolar fluids. In Theory and Applications; Birkhaüser: Boston, MA, USA, 1999.

12. Boldrini, J.L.; Rojas-Medar, M.A.; Fernández-Cara, E. Semi-Galerkin approximation and strong solutions to the equations of the nonhomogeneous asymmetric fluids. J. Math. Pures Appl. 2003, 82, 1499-1525. [CrossRef]

13. Braz e Silva, P.; Cruz, F.W.; Loayza, M.; Rojas-Medar, M.A. Global unique solvability of nonhomogeneous asymmetric fluids: A Lagrangian approach. J. Differ. Equ. 2020, 269, 1319-1348. [CrossRef]

14. Braz e Silva, P.; Cruz, F.W.; Rojas-Medar, M.A. Semi-strong and strong solutions for variable density asymmetric fluids in unbounded domains. Math. Methods Appl. Sci. 2017, 40, 757-774. [CrossRef]

15. Ferreira, L.C.F.; Villamizar-Roa, E.J. Micropolar fluid system in a space of distributions and large time behavior. J. Math. Anal. Appl. 2007, 332, 1425-1445. [CrossRef]

16. Villamizar-Roa, E.J.; Rodríguez-Bellido, M.A. Global existence and exponential stability for the micropolar fluid system. Z. Agew. Math. Phys. 2008, 59, 790-809. [CrossRef]

17. Prodi, G. Un teorema di unicità per le equazioné di Navier-Stokes. Ann. Mat. Pura Appl. 1959, 48, 173-182. [CrossRef]

18. Serrin, J. On the interior regularity of weak solutions of the Navier-Stokes equations. Arch. Ration. Mech. Anal. 1962, 9, 187-195. [CrossRef]

19. Ortega-Torres, E.; Rojas-Medar, M.A. On the regularity for solutions of the micropolar fluid equations. Rend. Semin. Mat. Univ. Padova 2009, 122, 27-37. [CrossRef]

20. Alghamdi, A.M.; Gala, S.; Kim, J.-M.; Ragusa, M.A. The anisotropic integrability logarithmic regularity criterion to the 3D micropolar fluid equations. AIMS Math. 2020, 5, 359-375. [CrossRef]

21. Gala, S.; Ragusa, M.A.; Théra, M. A new regularity criterion of weak solutions to the 3D micropolar fluid flows in terms of the pressure. Boll. Unione Mat. Ital. 2020. [CrossRef]

22. Gala, S.; Ragusa, M.A. A regularity criterion for 3D micropolar fluid flows in terms of one partial derivative of the velocity. arXiv 2020, arXiv:2005.004458v1.

23. Loayza, M.; Rojas-Medar, M.A. A weak $L^{p}$ - Prodi-Serrin type regularity criterion for the micropolar fluid equations. J. Math. Phys. 2016, 57, 021512. [CrossRef]

24. Ragusa, M.A.; Wu, F. A regularity criterion for three-dimensional micropolar fluid equations in Besov spaces of negative regular indices. Anal. Math. Phys. 2020, 30, 11. [CrossRef]

25. Ferreira, L.C.F.; Planas, G.; Villamizar-Roa, E.J. On the nonhomogeneous Navier-Stokes system with Navier friction boundary conditions. SIAM J. Math. Anal. 2013, 45, 2576-2595. [CrossRef]

26. Mulone, G.; Salemi, F. On the existence of hydrodynamic motion in a domain with mixed boundary type condition. Meccanica 1983, 18, 136-144. [CrossRef]

27. Mulone, G.; Salemi, F. On the hydrodynamic motion in a domain with mixed boundary conditions: Existence, uniqueness, stability and linearization principle. Anal. Mat. Pura Appl. 1985, 139, 147-174. [CrossRef]

28. Shimada, R.; Yamaguchi, N. On an existence theorem for the Navier-Stokes equations with free slip boundary condition in exterior domain. In Parabolic and Navier-Stokes Equations; Banach Center Publ., 81, Part. 2; Polish Academy of Sciences, Institute of Mathematics: Warsaw, Poland, 2008.

29. Amrouche, C.; Rejaiba, A. $L_{p}$-theory for Stokes and Navier-Stokes equations with Navier boundary conditions. J. Differ. Equ. 2014, 256, 1515-1547. [CrossRef]

30. Solonnikov, V.A.; Šcadilov, V.E. A certain boundary value problem for the stationary system of Navier-Stokes equations. Trudy Mat. Inst. 1973, 125, 196-210. 
31. Mallea-Zepeda, E.; Lenes, E.; Rodríguez Zambrano, J. Bilinear optimal control problem for the stationary Navier-Stokes equations with variable density and slip boundary condition. Bull. Braz. Math. Soc. 2019, 50, 871-887. [CrossRef]

32. Mallea-Zepeda, E.; Ortega-Torres, E.; Villamizar-Roa, E.J. A boundary control problem for micropolar fluids. J. Optim. Theory Appl. 2016, 169, 349-369. [CrossRef]

33. Mallea-Zepeda, E.; Ortega-Torres, E.; Villamizar-Roa, E.J. An optimal control problem for the steady nonhomogeneous asymmetric fluids. Appl. Math. Optim. 2019, 80, 299-329. [CrossRef]

34. Baranovskii, E.S. Global solutions for a model of polymeric flows with wall slip. Math. Methods Appl. Sci. 2017, 40, 5035-5043. [CrossRef]

35. Temam, R. Navier-Stokes equations. In Theory and Numerical Analysis; AMS Chelsea Publication: Providence, RI, USA, 2001.

36. Alekseev, G.V. Solvability of stationary boundary control problems for heat convection equations. Sib. Math. J. 1998, 39, 982-998. [CrossRef]

37. Lions, J.L. Quelques Métodes de Résolution des Problèmes Aux Limites Non Linéares. In Etudes Mathématiques; Dunod: Paris, France, 1969.

38. Simon, J. Compact sets in the space $L^{p}(0, T ; B)$. Ann. Mat. Pura Appl. 1987, 146, 65-96. [CrossRef]

39. Nirenberg, L. On elliptic partial differential equations. Ann. Scuola Norm. Sup. Pisa Cl. Sci. 1959, 13, 115-162. 